\title{
Sympathectomized tumor-bearing mice survive longer but develop bigger melanomas
}

\author{
${ }^{1}$ Horvathova L, ${ }^{1}$ Tillinger A, ${ }^{1,2}$ Padova A, ${ }^{1,2}$ Mravec B \\ ${ }^{1}$ Institute of Experimental Endocrinology, Biomedical Research Center, Slovak Academy of Sciences, Bratislava, Slovakia; \\ ${ }^{2}$ Institute of Physiology, Faculty of Medicine, Comenius University, Bratislava, Slovakia \\ E-mail: ueenlack@savba.sk
}

Objectives. Previously we have shown that 20 days after the tumor cells injection smaller melanomas have been developed in chemically sympathectomized mice in comparison with animals having intact sympathetic nervous system. However, it is known that chemical sympathectomy reduces the sympathetic neurotransmission only temporarily. In the present study, we monitored the survival of the sympathectomized mice with melanoma with an attempt to find out how long the suppressing effect of sympathectomy on the melanoma growth may endure.

Methods. The chemical sympathectomy was performed by intraperitoneal injection of neurotoxin 6-hydroxydopamine in male C57BL/6J mice. Seven days later, the animals were injected subcutaneously with B16-F10 melanoma cells. Then, melanoma development, survival of the tumorbearing mice and weight of the developed tumor mass were analyzed.

Results. Sympathectomy delayed the development of the palpable tumors (18th day vs.14th day) and significantly prolonged the survival of the tumor-bearing mice (median 34 days vs. 29 days). However, the weight of the developed melanoma was significantly increased in the sympathectomized mice in comparison with the animals having intact sympathetic nervous system.

Conclusions. The data of the present study showed that effect of the chemical sympathectomy, performed before the tumor growth induction, persisted even at the time when sympathetic nerves started to regenerate that resulted in a prolonged survival of the mice with melanoma. However, comparing to our previous study, in which we have shown a reduced tumor mass in earlier stages of the tumor growth, specifically 20 days after melanoma cells injection, now we indicate that in later stages of the melanoma progression, the tumor mass was significantly increased in sympathectomized animals. These contra-intuitive findings may indicate that interventions affecting the sympathetic nervous system may exert complex effect on the tumor progression. Based on these data we may suggest that the potential therapeutic interventions affecting the sympathetic signaling in the tumor tissue and its microenvironment should attenuate the sympathetic neurotransmission not only temporarily but till the complete regression of the tumor tissue.

Key words: 6-hydroxydopamine, B16-F10 melanoma cells, melanoma development and growth, tumor weight

Corresponding author: Lubica Horvathova, Ph.D., Institute of Experimental Endocrinology, Biomedical Research Center, Slovak Academy of Sciences, Dubravska cesta 9, 84505 Bratislava, Slovakia; phone: +421-2-32295223; e-mail: ueenlack@savba.sk. 
Experimental and clinical studies have shown that exposure to stress may affect several crucial processes related to the tumor growth, e.g. potentiate the proliferation and migration of the cancer cells, inhibit the tumor cell apoptosis, increase the tumor cell invasiveness and metastatic activity, support the neoangiogenesis in the tumor tissue, or suppress the natural killer cell activity (Godbout and Glaser 2006; Sood et al. 2006; Thaker et al. 2006; Andersen et al. 2007; Sephton et al. 2009; Hassan et al. 2013; Nagaraja et al. 2013). These effects are mediated especially by neurotransmitters released by sympathetic nerve endings, particularly by norepinephrine (Yang 2010). Norepinephrine may exert stimulatory effect on tumor growth by affecting the tumor microenvironment (e.g. suppressing the anti-tumor immunity and stimulating the vascular endothelial growth factor - VEGF) or binding directly on $\beta$-adrenergic receptors expressed by tumor cells with a consequent exaggeration of tumor cells proliferation and migration (Cole and Sood 2012). Moreover, neuropeptide Y, a co-transmitter released from the sympathetic nerve endings, may exert stimulatory effect on the tumor growth as well (Tilan and Kitlinska 2010).

Stimulatory effect of the sympathetic nervous system on the tumor growth and development of metastasis has been further supported by experimental and clinical studies, in which sympathetic neurotransmission was attenuated or eliminated (Raju et al.
2009; Sloan et al. 2010; Barron et al. 2011; Lackovicova et al. 2011; Magnon et al. 2013). Based on these facts, it has been suggested that interventions reducing the effect of the sympathetic neurotransmitters may represent a potential adjuvant therapy in oncological patients (Schuller 2010; Powe and Entschladen 2011; Tang et al. 2013). These interventions may be useful also for the secondary micro metastasis development prevention after surgical extirpation of the primary tumor mass (Glasner et al. 2010; Neeman et al. 2012). However, there is no consensus when exactly to start these interventions in oncological patients and how long it is necessary to attenuate sympathetic signalization in tumor tissue.

Previously we have shown that chemical sympathectomy, induced by 6-hydroxydopamine (6-OHDA) before tumor cells injection, significantly reduced the tumor mass in mice sacrificed 20 days after the injection of melanoma cells (Horvathova et al. 2016). However, it is known that approximately within one month after 6-OHDA administration, the sympathetic nerve endings start to regenerate (Kruszewska et al. 1995). Therefore, in this study we investigated the long-term effect of chemical sympathectomy, performed before tumor growth induction, on the melanoma progression. Hence, sympathectomised mice were injected with melanoma cells and development of tumors, survival of tumor-bearing mice, and weight of developed melanomas, were analyzed.

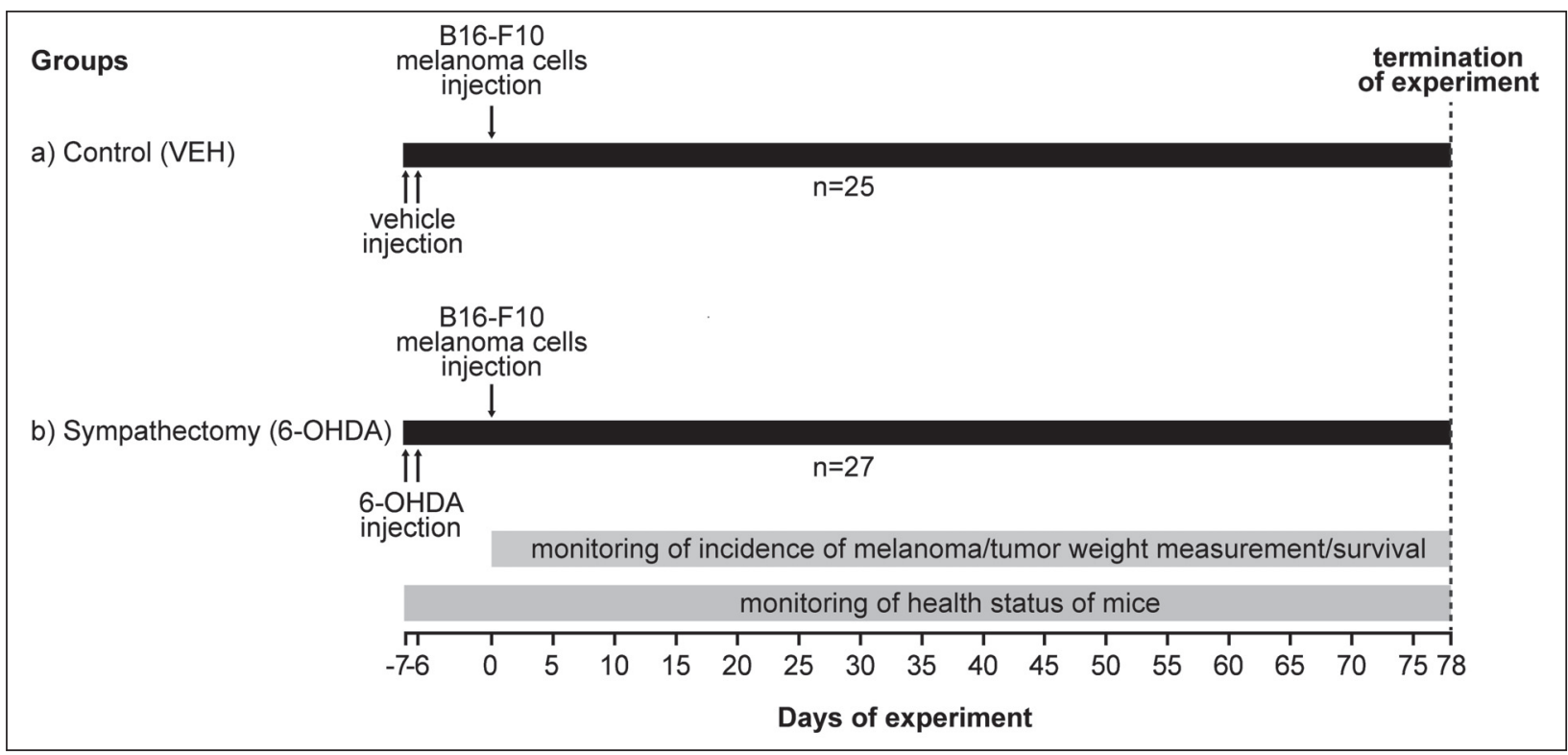

Figure 1. Schematic illustration of the experimental design. Abbreviations: 6-OHDA - 6-hydroxydopamine, VEH vehicle 


\section{Material and Methods}

Animals. The experiment was performed on 3 months-old male C57BL/6J mice. The mice, bred at the Institute of Experimental Endocrinology, were housed three to six per cage and kept under controlled conditions in the animal facility $(12 \mathrm{~h}$ light/dark cycle, lights on at 06:00 AM; temperature $22 \pm 2{ }^{\circ} \mathrm{C}$, humidity $55 \pm 10 \%$ ) with free access to tap water and standard pelleted chow. The weight of mice at the beginning of experiment was in the range of 24-27g. All experimental procedures were performed between 08:00-13:00 $\mathrm{h}$ to avoid the animals of all the external stressogen stimuli and were approved by the Animal Care Committee of the Institute of Experimental Endocrinology, Slovak Academy of Sciences, Bratislava, Slovakia and State Veterinary and Food Administration of the Slovak Republic (Approval No. Ro 1632/13-221). The animals received care in compliance with the Guide for the Care and Use of Laboratory Animals published by the U.S. National Institutes of Health.

Experimental protocol. Mice were randomly divided into two experimental groups based on the type of treatment (Figure 1): a) control mice with intact sympathetic nervous system injected later with tumor cells (VEH, $\mathrm{n}=25)$; b) sympathectomized mice injected later with tumor cells (6-OHDA, $n=27)$.

Chemical sympathectomy was performed seven days before melanoma cells inoculation in conscious mice by intraperitoneal injection of neurotoxin 6-hydroxydopamine hydrobromide (6-OHDA, $100 \mathrm{mg} / \mathrm{kg}$ of body weight, Sigma-Aldrich, Germany) over two consecutive days (Figure 1). The 6-OHDA was dissolved in a sterile saline containing $0.1 \%$ of the antioxidant ascorbic acid (Sigma-Aldrich, Germany). This dose has been shown to induce destruction of sympathetic nerve endings after 3-5 days and this effect lasted for at least 21 days in spleen (Kruszewska et al. 1995). Mice of VEH group were intraperitoneally injected with vehicle (sterile saline containing $0.1 \%$ of the antioxidant ascorbic acid). The efficiency of the sympathectomy was confirmed immediately after application of 6-OHDA by presence of ptosis in sympathectomized mice (Claude Bernard-Horner's syndrome) and blood in urine indicating the destruction of the sympathetic nerve endings in the urinary tract. Moreover, to confirm the efficiency of the sympathectomy, we determined norepinephrine concentrations in the spleen seven days after 6-OHDA administration (VEH: $2300.2 \pm 200.7$ vs. 6 -OHDA: $200.2 \pm 24.5 \mathrm{pg} / \mathrm{mg}$ of tissue; Figure 2).
One week after the administration of 6-OHDA or vehicle, all the mice were inoculated with the B16-F10 melanoma cells (Figure 1). A B16-F10 melanoma cell suspension was injected subcutaneously as a single dose of $3 \times 10^{3}$ tumor cells in $0.1 \mathrm{ml}$ of RPMI 1640 medium per mice without anesthesia. Before tumor cells inoculation, back of mice was shaven and disinfected. During the experiment, the health status of animals was monitored and mice were inspected daily for the presence of palpable tumor mass in the place of melanoma cells injection.

The mice of both the experimental groups were kept to determine the effect of the sympathetic denervation on the tumor incidence, progression, and survival of the tumor-bearing mice. The euthanasia time of these mice was determined based on their health status, especially the motor skills of mice and their capability to ingest were monitored. The last mouse with developed melanoma was euthanized on $78^{\text {th }}$ day after tumor cells injection. In two mice (from 6-OHDA group) no tumors developed. They were euthanized on the $78^{\text {th }}$ day and examined for the presence of melanoma to confirm the lack of tumor formation.

Statistical analysis. All statistical analyses were performed using SigmaPlot 11.0 (Systat Software,

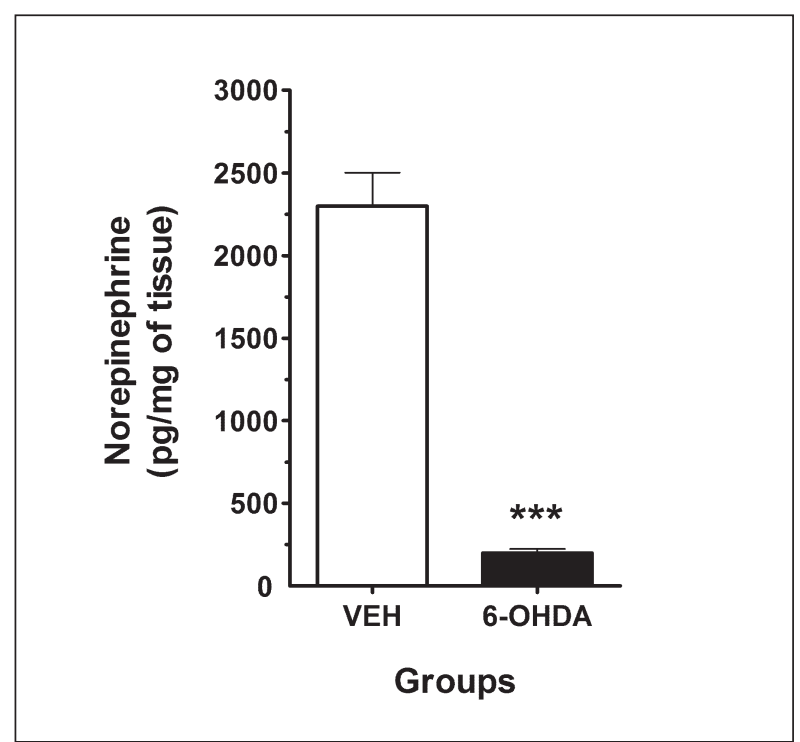

Figure 2. The effect of the chemical sympathectomy on the splenic norepinephrine concentration. Mice were injected with either 6-OHDA (6-OHDA; $\mathbf{\square}$; $=4$ ) or an equal volume of vehicle (VEH; $\square ; n=4)$. Concentration of splenic norepinephrine was determined by ELISA assay. Each value is the mean \pm SEM. ${ }^{* * *} \mathrm{p}<0.001$. Abbreviation: 6-OHDA - 6-hydroxydopamine, VEH - vehicle 
Inc., Germany). Differences between the groups were determined by Student t-test. Survival curves were analyzed according to Kaplan-Meier and the Log-rank test was used to determine differences in survival between groups. The results are expressed as means \pm SEM and represent an average of 25-27 animals. A value of $\mathrm{p}<0.05$ was considered statistically significant.

\section{Results}

Effect of sympathetic denervation on melanoma progression. In both experimental groups of the tumor-bearing mice (VEH and 6-OHDA), the first palpable tumors were observed on the $7^{\text {th }}$ day (Figure 3A). In the VEH group of mice, tumors were developed on the $14^{\text {th }}$ day in all the animals injected
A

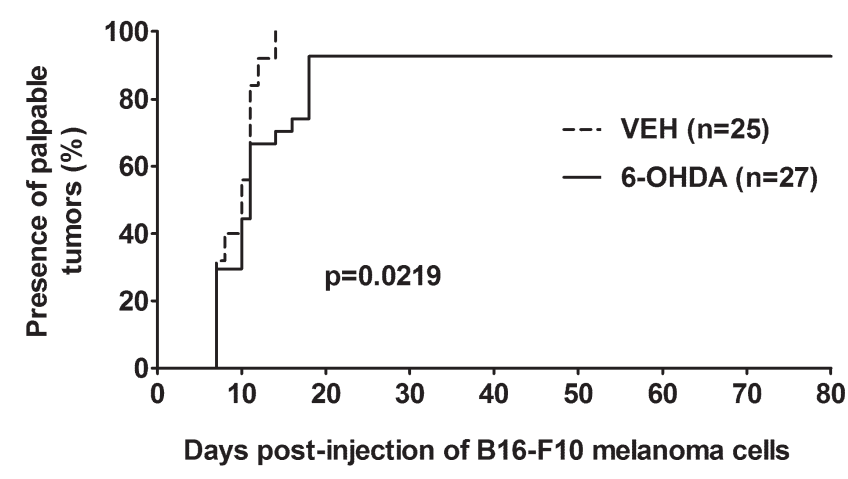

C

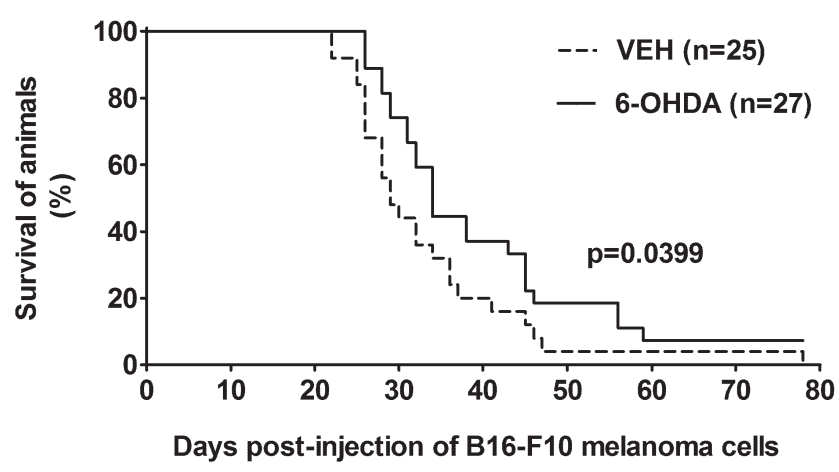

B

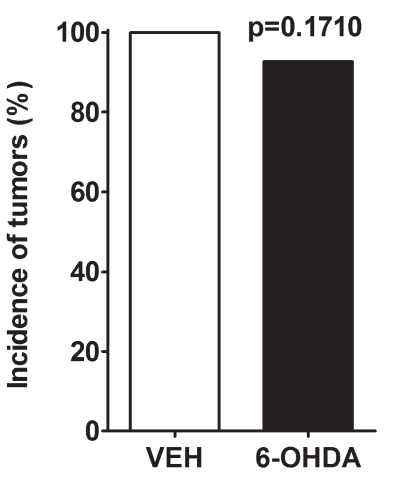

D

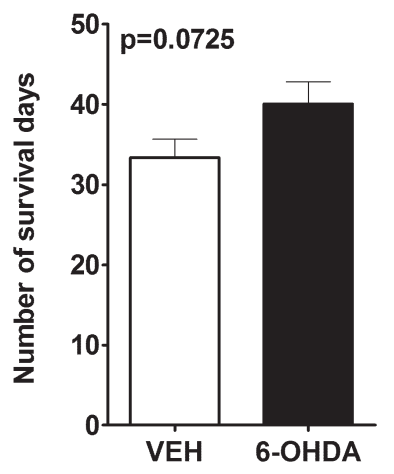

Figure 3. Effect of the chemical sympathectomy on the melanoma development and survival of the tumor-bearing mice. Percentage of the developed tumors in the sympathectomized mice (6-OHDA; -; $n=27)$ and mice with the intact sympathetic nervous system (VEH; ---; $\mathrm{n}=25)$ injected by B16-F10 melanoma cells $(\mathbf{A})$. Incidence of melanoma (\%) after injection of B16-F10 melanoma cells in sympathectomized mice (6-OHDA; $\mathbf{\square}$; $\mathrm{n}=27$ ) and mice with intact sympathetic nervous system (VEH; $\square ; n=25)(B)$. Survival of the sympathectomized mice (6-OHDA; -; n=27) and mice with intact sympathetic nervous system (VEH; ---; $\mathrm{n}=25$ ) injected by tumor cells $(\mathrm{C})$. Number of the survival days of the sympathectomized mice (6-OHDA; $\mathbf{\square}$ n=25) and mice with the intact sympathetic nervous system (VEH; $\square ; n=25)$ injected by B16-F10 melanoma cells (D). Value reflecting the number of survival days is expressed as the mean \pm SEM and each value represents an average of 25-27 mice (Log-rank test, Student t-test). Abbreviations: 6-OHDA - 6-hydroxydopamine, VEH - vehicle 
with melanoma cells (100\% incidence of tumors; Figure $3 \mathrm{~B}$ ). In 6-OHDA group of mice, two mice injected with melanoma cells did not show tumor development $(92.59 \%$ incidence of tumors; Figure $3 \mathrm{~B})$ and the last tumors appeared on the $18^{\text {th }}$ day post-injection of B16-F10 melanoma cells. Significantly delayed development of palpable tumors was observed in sympathectomized mice $(p=0.0219$; Figure 3A). Moreover, chemical sympathectomy also significantly prolonged the survival of mice ( $p=0.0399$; median for $\mathrm{VEH}: 29$ days, median for 6-OHDA: 34 days; Figure 3C). However, average number of survival days was not affected by sympathectomy (VEH: 33.36 \pm 2.292 ; 6-OHA: 40.04 \pm 2.784 ; $\mathrm{t}=1.835, \mathrm{df}=50, \mathrm{p}=0.0725$; Figure $3 \mathrm{D}$ ).

Finally, the effect of the chemical sympathectomy on melanoma progression was assessed also by weighting of the developed melanoma mass. The weight of the developed melanoma tissue was significantly increased in sympathectomized mice in comparison with animals from the $\mathrm{VEH}$ group $(t=2.424, \mathrm{df}=48, \mathrm{p}=0.0192$; Figure 4$)$.

\section{Discussion}

The aim of the present study was to find out whether temporal elimination of sympathetic neurotransmission, performed before tumor growth induction, may exert a long-lasting effect on the progression of melanoma in mice. We observed that chemical sympathectomy resulted not only in a later development of tumors, but also significantly prolonged the survival of these animals compared to mice with an intact sympathetic neurotransmission. Interestingly, the weight of the melanomas was significantly higher in sympathectomized mice than in mice with intact sympathetic nervous system.

Clinical and experimental data have shown that sympathetic nervous system may significantly potentiate the cancer progression. In support of this assumption, women with breast cancer that underwent psychological interventions reducing stress or were treated by $\beta$-blockers for hypertension, survived longer when compared to matched controls (GieseDavis et al. 2011). Interventions affecting the sympathetic neurotransmission in tumor-bearing animals (e.g. sympathectomy, administration of $\beta$-blockers) have shown stimulatory effect of sympathetic nervous system on several experimental tumors, as well. For example, chemical sympathectomy performed by 6-OHDA attenuated the incidence of N-methyl- $\mathrm{N}^{\prime}$ nitro-N-nitrosoquanidine (MNNG) induced gastric cancer in rats (Tatsuta et al. 1992), inhibited the

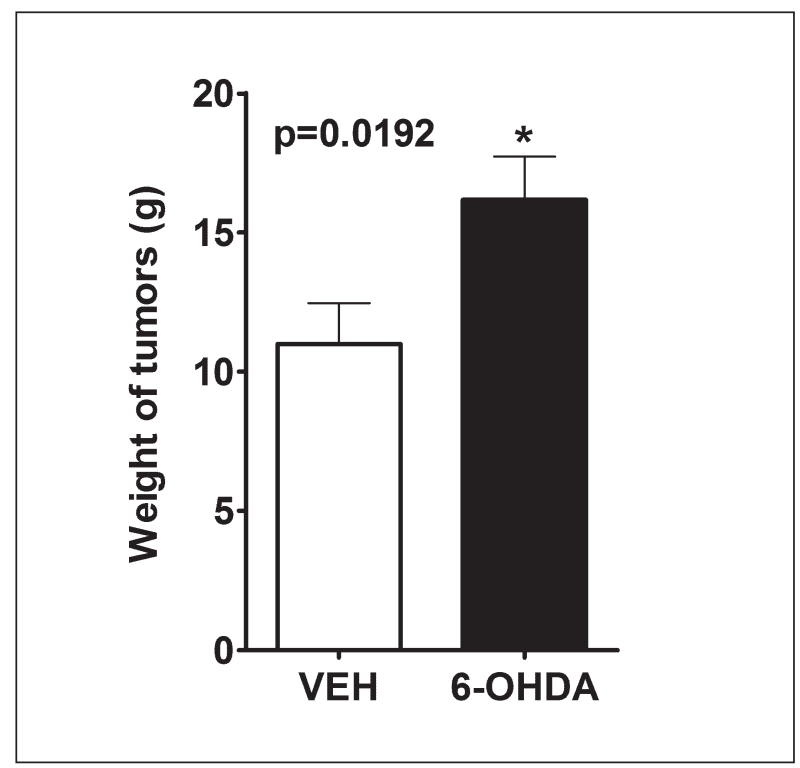

Figure 4. Tumor weight (g) in the tumor-bearing mice with the intact sympathetic nervous system (VEH; $\square$; $\mathrm{n}=25)$ and the sympathectomized tumor-bearing mice (6-OHDA; $\boldsymbol{\square} ; n=25)$ in survival study. Each value is expressed as the mean \pm SEM and represents an average of 25 mice. Statistical significance compared with VEH group: ${ }^{\star} \mathrm{p}<0.05$ (Student $t$-test). Abbreviations: 6-OHDA -6-hydroxydopamine, VEH - vehicle

growth pattern of LPC-1 plasmacytoma tumors in mice (Grzanna et al. 1985), and also decreased the incidence of intra-abdominal fibrosarcoma tumors in rats and prolonged their survival (Lackovicova et al. 2011). Similarly, bilateral surgical sympathectomy in rats resulted in the development of significantly smaller and less invasive tongue tumors than in sham-operated animals (Raju et al. 2007; Raju et al. 2009). Moreover, pre-treatment of tumor-bearing rats with $\beta$-blockers significantly reduced the development of tumors in stressed animals as well as development of metastases after surgical removal of the tumor mass (Sloan et al. 2010). Unilateral superior cervical ganglionectomy in mice with subcutaneous mammary tumor masses in an ear also attenuated the tumor growth approximately by more than $50 \%$ compared with the contralateral tumors induced in ear with intact sympathetic innervation (Romeo et al. 1991). In their complex study, Magnon and co-workers (2013) have tested both type of sympathectomy and have found that the early phases of the prostate cancer development were prevented by chemical as well as surgical sympathetic denervations (Magnon et al. 2013). However, it is necessary to note that not all the studies reported have attenuated the tumor 
growth under conditions of reduced sympathetic transmission. However, the number of these studies is only sparse (Brenner et al. 1992; Basu et al. 2004).

The effect of eliminated or attenuated sympathetic neurotransmission on cancer progression and metastasis formation is mediated by several mechanisms. Basu with co-workers (2004) have attributed an important role of dopamine in markedly increased growth of malignant B16 melanoma tumors in mice and suggested that dopamine might regulate malignant tumor growth by modulating angiogenesis, since blood vessels are supplied by peripheral dopaminergic nerves. On the other hand, Raju and colleagues (2007) have explained the cause of significantly smaller and less invasive tongue tumors in bilaterally sympathectomized rats by the differences in the sympathetic innervation pattern within and around the tumors and decreased interstitial fluid pressure and lymph vessel area. In another study of Raju with collective (2009), have found that sympathetic denervation significantly may alter the geneexpression profile of tumor cells. They have found 280 genes differentially expressed in tumors with intact sympathetic innervation compared with the bilaterally sympathectomized tumors. Alterations have been found in genes associated with the cell adhesion and signalling structure, proliferation, metabolism, lymphangiogenesis, growth and development, and immunity (Raju et al. 2009). Similarly, we also found in melanoma tissue changes in expression profile of many genes related to cancer-limiting processes, such as apoptosis, hypoxia, and angiogenesis, induced by chemical denervation in mice (Horvathova et al. 2016).

In our previous study, we have shown that chemical sympathectomy may significantly reduce the mass of the melanoma tissue in mice sacrificed 20 days after the injection of tumor cells (Horvathova et al. 2016). However, it is known that chemical sympathectomy exerts only a temporal destruction of sympathetic nerve endings. Within approximately one month these nerve endings start to regenerate (Kruszewska et al. 1995) and sympathetic neurotransmission may be even exaggerated (our data, not published). Therefore, in the present study, we investigated whether chemical sympathectomy may exert long-lasting effect on tumor growth. We are demonstrating a beneficial effect of the elimination of the sympathetic neurotransmission on the development of melanoma and survival in mice. However, surpris- ingly, we observed that mass of melanoma tissue was increased in the sympathectomised mice kept for monitoring of survival. We propose that this survival might be a consequence of the noradrenergic nerve endings regeneration, which begins already 21 days after 6-OHDA application (Kruszewska et al. 1995). This finding may reflect also activation of a complex adaptation or compensation responses within the tumor microenvironment deprived of the sympathetic neurotransmitters (e.g. norepinephrine, neuropeptide Y).

Based on the above-mentioned findings, we propose that attenuation of the sympathetic neurotransmission may represent a potent adjuvant therapeutic approach for the oncological patients. However, for the inhibition of tumor growth, it will be necessary to maintain the reduced sympathetic neurotransmission as long as possible. Reduced transmission of signals from sympathetic nerve endings to the cells effectors (e.g. tumor cells, immune cells, fibroblasts) may be utilized for blockade of the effects of the psychological stress that accompanies the cancer diagnosis as well as for the attenuation of the secondary metastasis development after surgical removal of the primary tumor mass (Andersen et al. 2007; Powe and Entschladen 2011; Neeman et al. 2012). However, it is necessary to determine: 1) when to start with the interventions reducing the sympathetic neurotransmission and 2) for how long these therapeutic approaches in the given patient should be used.

In conclusion, experimental and clinical findings support the assumption that reduction of the signalization related to sympathetic neurotransmission (e.g. administration of $\beta$-blockers, systemic or local sympathetic denervation of tumor tissues) may represent an adjuvant therapeutical approach for the oncological patients. However, tumor tissue represents highly complex system that rapidly responses to any treatment interventions (Holohan et al. 2013) and it can be expected that reduced sympathetic neurotransmission in the tumor microenvironment may induce several (adaptive) responses at the level of the tumor tissue and the level of the cancer cells.

\section{Acknowledgments}

This work was supported by the Slovak Research and Development Agency No. APVV-0007-10 and VEGA grant No. 2/0028/16.

\section{References}

Andersen BL, Farrar WB, Golden-Kreutz D, Emery CF, Glaser R, Crespin T, Carson WE, $3^{\text {rd }}$. Distress reduction from a psychological intervention contributes to improved health for cancer patients. Brain Behav Immun 21, 953-961, 2007. 
Barron TI, Connolly RM, Sharp L, Bennett K, Visvanathan K. Beta blockers and breast cancer mortality: a population-based study. J Clin Oncol 29, 2635-2644, 2011.

Basu S, Sarkar C, Chakroborty D, Nagy J, Mitra RB, Dasgupta PS, Mukhopadhyay D. Ablation of peripheral dopaminergic nerves stimulates malignant tumor growth by inducing vascular permeability factor/vascular endothelial growth factor-mediated angiogenesis. Cancer Res 64, 5551-5555, 2004.

Brenner GJ, Felten SY, Felten DL, Moynihan JA. Sympathetic nervous system modulation of tumor metastases and host defense mechanisms. J Neuroimmunol 37, 191-201, 1992.

Cole SW, Sood AK. Molecular pathways: beta-adrenergic signaling in cancer. Clin Cancer Res 18, 1201-1206, 2012.

Giese-Davis J, Collie K, Rancourt KM, Neri E, Kraemer HC, Spiegel D. Decrease in depression symptoms is associated with longer survival in patients with metastatic breast cancer: a secondary analysis. J Clin Oncol 29, 413-420, 2011.

Glasner A, Avraham R, Rosenne E, Benish M, Zmora O, Shemer S, Meiboom H, Ben-Eliyahu S. Improving survival rates in two models of spontaneous postoperative metastasis in mice by combined administration of a betaadrenergic antagonist and a cyclooxygenase-2 inhibitor. J Immunol 184, 2449-2457, 2010.

Godbout JP, Glaser R. Stress-induced immune dysregulation: implications for wound healing, infectious disease and cancer. J Neuroimmune Pharmacol 1, 421-427, 2006.

Grzanna R, Frondoza CG, Otten U. Sympathectomy inhibits growth of a murine plasmacytoma tumor. J Auton Nerv Syst 13, 149-160, 1985.

Hassan S, Karpova Y, Baiz D, Yancey D, Pullikuth A, Flores A, Register T, Cline JM, D’Agostino R, Jr., Danial N, Datta SR, Kulik G. Behavioral stress accelerates prostate cancer development in mice. J Clin Invest 123, 874-886, 2013.

Holohan C, Van Schaeybroeck S, Longley DB, Johnston PG. Cancer drug resistance: an evolving paradigm. Nat Rev Cancer 13, 714-726, 2013.

Horvathova L, Padova A, Tillinger A, Osacka J, Bizik J, Mravec B. Sympathectomy reduces tumor weight and affects expression of tumor-related genes in melanoma tissue in the mouse. Stress 19, 528-534, 2016.

Kruszewska B, Felten SY, Moynihan JA. Alterations in cytokine and antibody production following chemical sympathectomy in two strains of mice. J Immunol 155, 4613-4620, 1995.

Lackovicova L, Banovska L, Bundzikova J, Janega P, Bizik J, Kiss A, Mravec B. Chemical sympathectomy suppresses fibrosarcoma development and improves survival of tumor-bearing rats. Neoplasma 58, 424-429, 2011.

Magnon C, Hall SJ, Lin J, Xue X, Gerber L, Freedland SJ, Frenette PS. Autonomic nerve development contributes to prostate cancer progression. Science 341, 1236361, 2013.

Nagaraja AS, Armaiz-Pena GN, Lutgendorf SK, Sood AK. Why stress is BAD for cancer patients. J Clin Invest 123, 558-560, 2013.

Neeman E, Zmora O, Ben-Eliyahu S. A new approach to reducing postsurgical cancer recurrence: perioperative targeting of catecholamines and prostaglandins. Clin Cancer Res 18, 4895-4902, 2012.

Powe DG, Entschladen F. Targeted therapies: Using beta-blockers to inhibit breast cancer progression. Nat Rev Clin Oncol 8, 511-512, 2011.

Raju B, Haug SR, Ibrahim SO, Heyeraas KJ. Sympathectomy decreases size and invasiveness of tongue cancer in rats. Neuroscience 149, 715-725, 2007.

Raju B, Hultstrom M, Haug SR, Ibrahim SO, Heyeraas KJ. Sympathectomy suppresses tumor growth and alters geneexpression profiles in rat tongue cancer. Eur J Oral Sci 117, 351-361, 2009.

Romeo HE, Colombo LL, Esquifino AI, Rosenstein RE, Chuluyan HE, Cardinali DP. Slower growth of tumours in sympathetically denervated murine skin. J Auton Nerv Syst 32, 159-164, 1991.

Sephton SE, Dhabhar FS, Keuroghlian AS, Giese-Davis J, McEwen BS, Ionan AC, Spiegel D. Depression, cortisol, and suppressed cell-mediated immunity in metastatic breast cancer. Brain Behav Immun 23, 1148-1155, 2009.

Schuller HM. Beta-adrenergic signaling, a novel target for cancer therapy? Oncotarget 1, 466-469, 201Sloan EK, Priceman SJ, Cox BF, Yu S, Pimentel MA, Tangkanangnukul V, Arevalo JM, Morizono K, Karanikolas BD, Wu $\mathrm{L}$, Sood AK, Cole SW. The sympathetic nervous system induces a metastatic switch in primary breast cancer. Cancer Res 70, 7042-7052, 2010.

Sood AK, Bhatty R, Kamat AA, Landen CN, Han L, Thaker PH, Li Y, Gershenson DM, Lutgendorf S, Cole SW. Stress hormone-mediated invasion of ovarian cancer cells. Clin Cancer Res 12, 369-375, 2006.

Tang J, Li Z, Lu L, Cho CH: beta-Adrenergic system, a backstage manipulator regulating tumour progression and drug target in cancer therapy. Semin Cancer Biol 23, 533-542, 2013. 
Tatsuta M, Iishi H, Baba M, Taniguchi H. Inhibitions by 6-hydroxydopamine and neostigmine singly or together of gastric carcinogenesis induced by N-methyl-N'-nitro-N-nitrosoguanidine in Wistar rats. Int J Cancer 51, 767-771, 1992.

Thaker PH, Han LY, Kamat AA, Arevalo JM, Takahashi R, Lu C, Jennings NB, Armaiz-Pena G, Bankson JA, Ravoori M, Merritt WM, Lin YG, Mangala LS, Kim TJ, Coleman RL, Landen CN, Li Y, Felix E, Sanguino AM, Newman RA, Lloyd M, Gershenson DM, Kundra V, Lopez-Berestein G, Lutgendorf SK, Cole SW, Sood AK. Chronic stress promotes tumor growth and angiogenesis in a mouse model of ovarian carcinoma. Nat Med 12, 939-944, 2006.

Tilan J, Kitlinska J. Sympathetic Neurotransmitters and Tumor Angiogenesis-Link between Stress and Cancer Progression. J Oncol 2010, 539706, 2010.

Yang EV. Role for catecholamines in tumor progression: possible use for beta-blockers in the treatment of cancer. Cancer Biol Ther 10, 30-32, 2010. 